DOI: 10.24835/1607-0763-2019-2-27-35

Робот-ассистированные чрескожные вмешательства под КТ-контролем: первый опыт

\author{
Буровик И.А. ${ }^{*}$ Прохоров Г.Г., Лушина П.А., Васильев А.В., Дегтярёва Е.А.
}

ФГБу “НМИЦ онкологии имени Н.Н. Петрова” Минздрава России, Санкт-Петербург, Россия

\title{
CT-guided robotic-assisted percutaneous interventions: first experience
}

\author{
Burovik I.A. * , Prohorov G.G. , Lushina P.A., Vasiliev A.V., Degtiareva E.A. \\ FSBI "N.N. Petrov NMRC of Oncology" Ministry of Healthcare of the Russian Federation, St. Petersburg, Russia
}

Компьютерная томография (КТ) является эффективным методом контроля проведения малоинвазивный чрескожных вмешательств. Наиболее часто для лучевого мониторинга используются последовательный режим КТ-сканирования и КТ-флюороскопия. Альтернативным и относительно новым способом контроля выполнения интервенционных процедур является применение роботизированных КТ-совместимых устройств.

Цель исследования: продемонстрировать удобство, эффективность и безопасность роботизированной пункции как способа выполнения интервенционных процедур под КТ-контролем.

Материал и методы. Операции проводились в условиях КТ-операционной ФГБУ “НМИЦ онкологии имени Н.Н. Петрова" Минздрава России с использованием компьютерного томографа Philips Ingenuity и роботизированной приставки Maxio Perfint. В статье представлены клинические наблюдения биопсии опухоли надпочечника и криоабляции опухоли почки. При выполнении криоабляции применялась Медицинская криотерапевтическая система.

Результаты. Использование роботизированного устройства позволило выполнить соответствующие манипуляции.

Заключение. Роботизированная приставка Махіо представляется перспективным техническим решением для КТ-контролируемых чрескожных интервенций. Оценка лечебной эффективности использования роботизированной приставки в сравнении с традиционными подходами КТ-контроля при миниинвазивных вмешательствах требует дальнейшего изучения и анализа на более объемной выборке в более длительные сроки наблюдения.

Ключевые слова: интервенции под КТ-контролем, миниинвазивные чрескожные вмешательства в онкологии, КТ-совместимые роботизированные системы, робот-ассистированные операции под КТ-контролем.

Ссылка для цитирования: Буровик И.А., Прохоров Г.Г., Лушина П.А., Васильев А.В., Дегтярёва Е.А.
Робот-ассистированные чрескожные вмешательства под КТ-контролем: первый опыт. Медицинская визуализация. 2019; 23 (2): 27-35.

DOI: 10.24835/1607-0763-2019-2-27-35.

$$
\text { *** }
$$

Computed tomography is an effective method of monitoring of percutaneous interventions. Conventional CT guidance and CT-fluoroscopy are used most frequently for procedure monitoring. Image-guided robotic needle positioning is an alternative and relatively modern approach for interventional procedures.

Purpose: to demonstrate convenience, effectiveness and safety of robotic system for CT-guided interventions.

Materials and methods. CT-guided percutaneous interventions were performed in FSBI "N.N. Petrov NMRC of Oncology" Ministry of Healthcare of The Russian Federation using the Philips Ingenuity CT scanner and Maxio Perfint robotic system. This article presents clinical observations of adrenal tumor biopsy and cryoablation of a renal cell carcinoma. For cryoablation the Medical Cryotherapeutic System was used.

Results. The robotic system for CT-guided interventions allowed to perform corresponding surgical procedures.

Conclusions. The Maxio robotic console seems to be a promising technical solution for CT-guided interventions. Evaluation of the effectiveness of the robotic and traditional approaches of CT control in percutaneous procedures requires further investigations and analysis on larger data sample in longer periods of observation.

Key words: CT-guided interventions, mini-invasive percutaneous procedures in oncology, CT-compatible robotic systems, CT-guided robotic-assisted interventions.

Recommended citation: Burovik I.A., Prohorov G.G., Lushina P.A., Vasiliev A.V., Degtiareva E.A. CT-guided robotic-assisted percutaneous interventions: first experience. Medical Visualization. 2019; 23 (2): 27-35. DOI: 10.24835/1607-0763-2019-2-27-35. 


\section{Введение}

Компьютерная томография (КТ) является эффективным методом контроля проведения малоинвазивных чрескожных стереотаксических вмешательств $[1,2]$. К наиболее часто выполняемым подобным операциям относятся биопсии, дренирования и различные виды абляции.

Как правило, для лучевого мониторинга интервенций используется или последовательный режим КТ, или режим КТ-флюороскопии. При первом варианте врач постепенно, шаг за шагом продвигает иглу в необходимом направлении и периодически, выходя из процедурной кабинета КТ, выполняет сканирования для контроля положения инструмента в пространстве. С учетом того, что при такой операции само продвижение иглы происходит "вслепую", имеется относительно больший риск повреждения соседних органов и других анатомических структур, кроме того, процедура занимает относительно много времени, высока лучевая нагрузка на пациента за счет многократных сканирований $[3,4]$. В то же время медицинский персонал в этой ситуации, как правило, не подвергается лучевому воздействию. В противоположность этому при интервенционном вмешательстве под КТ-флюороскопией врач проводит операцию в режиме «реального времени», стоя около апертуры гентри в зоне воздействия ионизирующего излучения. Такой подход обеспечивает лучший уровень визуального контроля, сокращает время операции и число послеоперационных осложнений, тем самым повышает эффективность процедуры [4, 5].

Залогом успеха интервенций под КТ-контролем является точность установки инструмента в зону интереса. Однако при проведении операции и в последовательном режиме, и способом КТфлюороскопии ограничивающими факторами могут явиться недостаточность пространства апертуры гентри для размещения инструмента и сложность достижения цели при значительном отклонении траектории иглы от осей у и z $[6,7]$.

Для решения проблем сложной траектории введения иглы, снижения лучевой нагрузки на врача и пациента, а в итоге для повышения эффективности и безопасности малоинвазивных чрескожных вмешательств активно разрабатываются КТсовместимые роботизированные стереоскопические системы $[8,9]$. Одним из таких технических решений является серийно выпускаемый аппарат этой направленности роботизированная приставка Maxio Perfint.

\section{Цель исследования}

Продемонстрировать удобство, эффективность и безопасность роботизированной пункции как способа выполнения интервенционных процедур под КТ-контролем.

\section{Материал и методы}

Малоинвазивные чрескожные вмешательства проводились в условиях КТ-операционной ФГБУ "НМИЦ онкологии имени Н.Н. Петрова" Минздрава России с использованием компьютерного томографа Philips Ingenuity и роботизированной приставки Maxio ${ }^{\circledR}$ Perfint. При выполнении криобляции опухоли почки применялась Медицинская криотерапевтическая система (МКC).

Основными функциональными элементами приставки являются (рис. 1):

- подвижная стойка. Устанавливается перед процедурой справа или слева от стола томографа на специальные металлические платформы. Внутри стойки размещены блоки управления и вспомогательные механизмы, а на ней - монитор и рука-манипулятор;

- монитор. На этапе планирования на экране производится выбор типа манипуляции: биопсия, абляция, анестезия, другое. При этом в случае выбора аблятивного вмешательства имеется возможность моделирования объема зоны воздействия и конфигурации формы предполагаемой зоны некроза, исходя из количества и взаиморасположения электродов/зондов при использовании оборудования различных производителей. Программное обеспечение позволяет провести сегментацию печени, в том числе при совмещении серий изображений нескольких фаз контрастирования. При проведении абляции можно запланировать одновременное введение до 6 электродов,

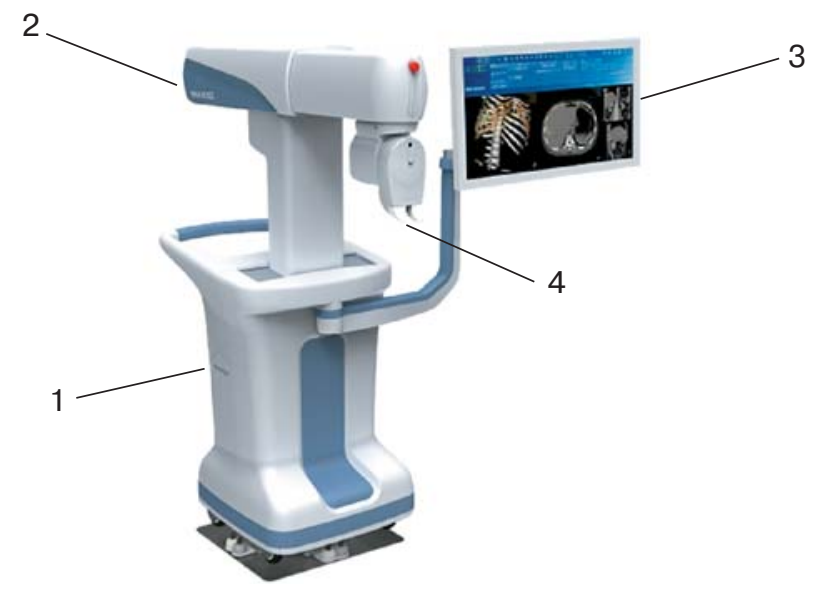

Рис. 1. Роботизированная приставка Мaxio ${ }^{\circledR}$ Perfint (внешний вид). 1 - стойка; 2 - рука-манипулятор; 3 монитор; 4 - пластина-фиксатор (стрелки).

Fig. 1. Maxio ${ }^{\circledR}$ Perfint robotic system (general appearance). 1 - base; 2 - robotic arm; 3 - monitor; 4 - end-effector of the robotic arm (arrows). 
при этом система автоматически рассчитывает оптимальный порядок их установки. Для контроля эффективности процедуры имеется возможность после выполнения контрольного КТ-сканирования на мониторе роботизированной приставки совместить до- и послеоперационные изображения и, таким образом, определить степень отклонения иглы от намеченной траектории или несовпадение размеров и формы зоны абляции относительно планируемых;

- рука-манипулятор. Представляет собой подвижный элемент роботизированной приставки. После этапа планирования рабочий элемент рукиманипулятора придвигается к поверхности тела пациента в проекции точки вкола и ориентируется в пространстве таким образом, чтобы обеспечить нужную траекторию введения инструмента. На конце "руки" находятся подвижные металлические "лапки", в которых зажимается специальная разъемная пластиковая проставка, имеющая внутри канал для иглы. Диаметр канала варьирует в зависимости от толщины инструмента от 11 до $21 \mathrm{G}$;

- пояс-индикатор для контроля дыхания. Вспомогательный элемент роботизированной системы, предназначенный для обеспечения одинаковой глубины вдоха пациента на различных этапах проведения операции. Необходимость подобного приспособления обусловлена тем, что при изменении объема легких может происходить значительное перемещение зоны интереса, расположенной в груди или животе. В то же время для точного позиционирования иглы критически важным является идентичность локализации цели при предоперационном сканировании и на этапе введении инструмента. Пояс фиксируется на уровне груди или живота и оснащен датчиками, определяющими степень его растяжения. Последняя отражается на шкале световых индикаторов, устанавливаемой в зоне видимости для пациента. При этом после небольшой тренировки больной, выполняя команды врача, может самостоятельно отслеживать нужную амплитуду дыхательных экскурсий;

- фиксирующий вакуумный матрас. Матрас внутри содержит мелкие легкие шарики из синтетического материала и имеет клапан для нагнетания/эвакуации воздуха. При проведении процедуры матрас размещается на столе томографа, далее на него укладывают пациента и свободными боковыми поверхностями матраса оборачивают его, оставляя свободной область оперативного доступа. Далее из камеры матраса с помощью компрессора откачивают воздух, при этом матрас, сохраняя приданную ему форму, прочно фиксируется, что препятствует движению пациента и в дальнейшем обеспечивает точность позиционирования иглы.

Для демонстрации роботизированной приставки приводим показательные клинические наблюдения: диагностическое (трепанбиопсия опухоли надпочечника) и лечебное (криоабляция опухоли почки).

\section{Результаты}

\section{Клиническое наблюдение 1}

Пациент М., 54 года, диагноз: "меланома кожи спины (р)TxN1bM0. Прогрессирование процесса после нерадикального лечения в 2017 г.: появление метастазов в мягкихтканяхшеи, затылочныхлимфоузлах. Состояние на фоне терапии пембролизумабом с 13.08.2018".

При контрольном обследовании после 4-го цикла терапии по данным КТ шеи, груди, живота отмечено появление образования в теле левого надпочечника размерами $25 \times 17$ мм; метастазы в затылочных лимфоузлах значимо в размерах не изменились, отмечен полный регресс метастазов в мягких тканях шеи. Эффект лечения расценен как псевдопрогрессирование. После 6-го цикла зарегистрировано дальнейшее увеличение образования в надпочечнике до $35 \times 33$ мм (рис. 2).

С учетом подозрения на прогрессирование опухолевого процесса с целью гистологической верификации изменений принято решение о выполнении трепанбиопсии образования надпочечника.

В связи с большой массой пациента и неудовлетворительной визуализацией опухоли при УЗИ было принято решение о проведении биопсии под КТ-контролем с применением роботизированной приставки. Как было описано выше, пациент был фиксирован с помощью матраса на столе томографа в положении "на животе", контроль глубины вдоха пациентом осуществлялся посредством специального пояса-индикатора. Проведено предоперационное КТ-сканирование (рис. 3).

Как видно на рис. 3, при доступе к опухоли надпочечника в аксиальной плоскости биопсийная игла с большой вероятностью пройдет через плевральную полость и легочную паренхиму, что может стать причиной развития осложнений - пневмо- или гемоторакса. В связи с этим оптимальным вариантом в этой ситуации является введение иглы под углом. Полученные КТ-данные загружены в роботизированную приставку, где выполнена предоперационная разметка траектории вкола иглы (рис. 4). Далее рука-манипулятор робота позиционирована над точкой вкола.

После проведения местной анестезии биопсийная игла введена через пластиковую проставку, фиксированную в лапках руки-манипулятора (рис. 5). Глубина введения иглы до образования составила 18 см.

Выполнено контрольное сканирование для определения положения иглы (рис. 6). Как видно на рисунке, 


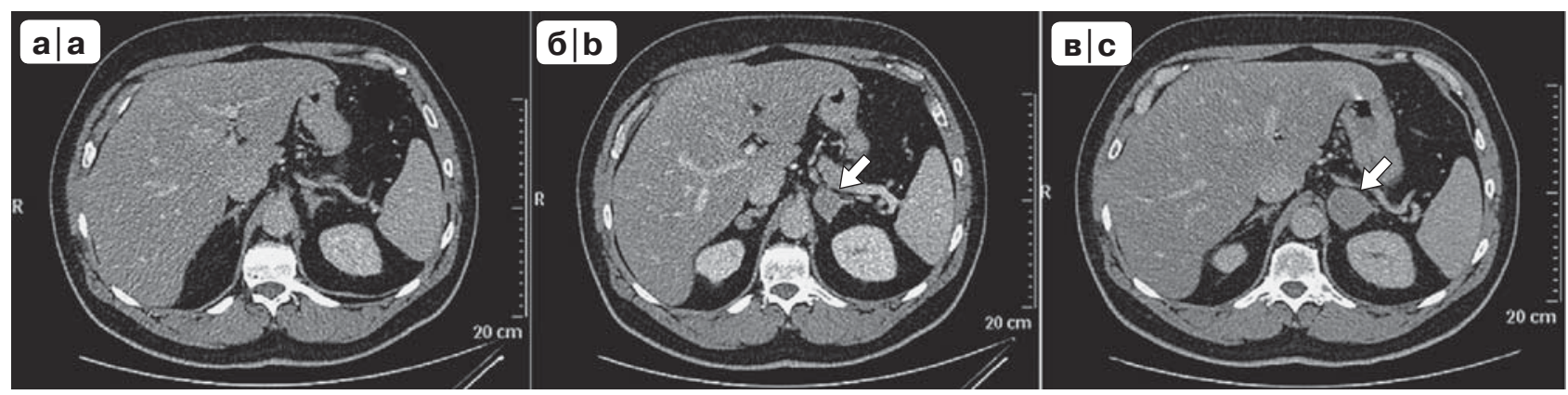

Рис. 2. Компьютерные томограммы брюшной полости пациента М., 54 лет. Образование в теле левого надпочечника (стрелки). а - до начала лечения пембролизумабом образование в надпочечнике не выявляется; б - через 4 цикла терапии пембролизумабом образование размерами $25 \times 17$ мм; в - через 6 циклов терапии пембролизумабом образование размерами $35 \times 33$ мм.

Fig. 2. Abdominal CT scan of the 54-year-old patient, M. The lesion in the body of the left adrenal gland (arrows). a - before starting pembrolizumab: no evidence of lesion in the adrenal gland; b - after 4 cycles of pembrolizumab: the lesion is $25 \times 17 \mathrm{~mm}$ in size; c - after 6 cycles of pembrolizumab: the lesion is $35 \times 33 \mathrm{~mm}$ in size.
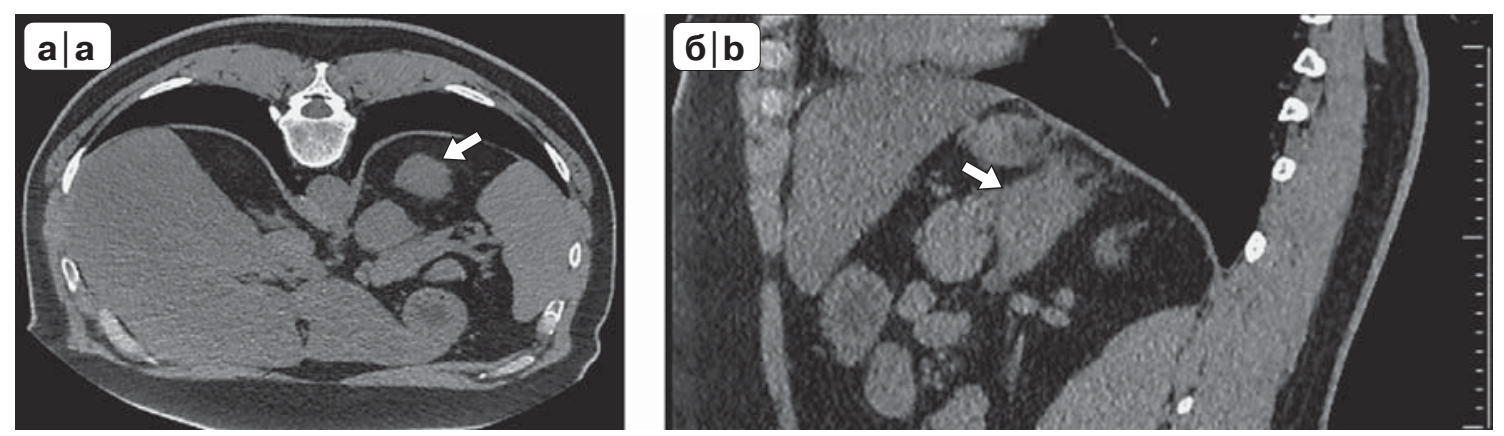

Рис. 3. Компьютерные томограммы брюшной полости пациента М., 54 лет, в положении на животе перед биопсией. a - аксиальный срез на уровне образования; б - MPR-реформация в сагиттальной плоскости. Образование в теле левого надпочечника (стрелки). Пояснение в тексте.

Fig. 3. Abdominal CT scan of the 54-year-old patient, $M$. in the prone position before biopsy. a - axial scan at the level of the tumor; b - MPR in sagittal plane. The lesion in the body of the left adrenal gland (arrows). The explanation is in the text.

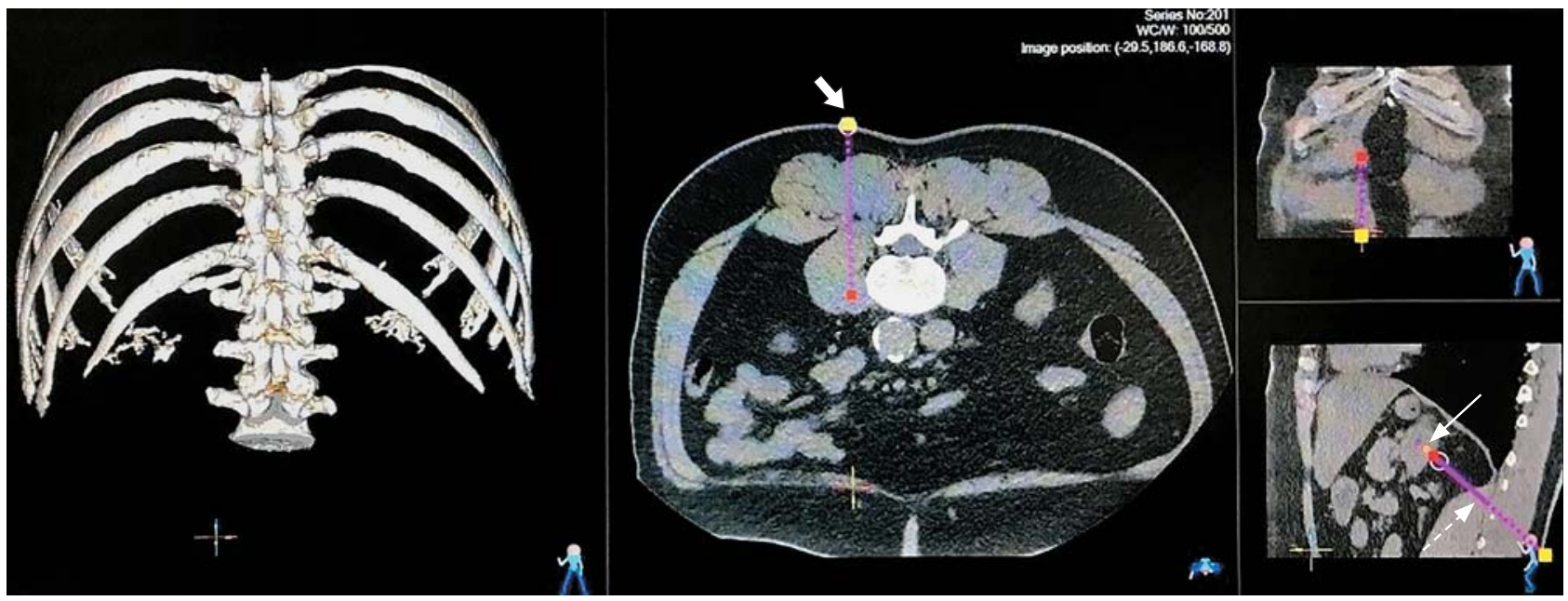

Рис. 4. Компьютерная томограмма пациента М., 54 лет, загруженная в роботизированную приставку Махіо для предоперационной разметки: точка вкола (толстая стрелка), точка цели (тонкая стрелка), планируемая траектория иглы (пунктирная стрелка).

Fig. 4. CT scan of the 54-year-old patient, M., which loaded into the Maxio robotic system for preoperative marking: the entry point (thick arrow), the target point (thin arrow), needle path planning (dashed arrow). 


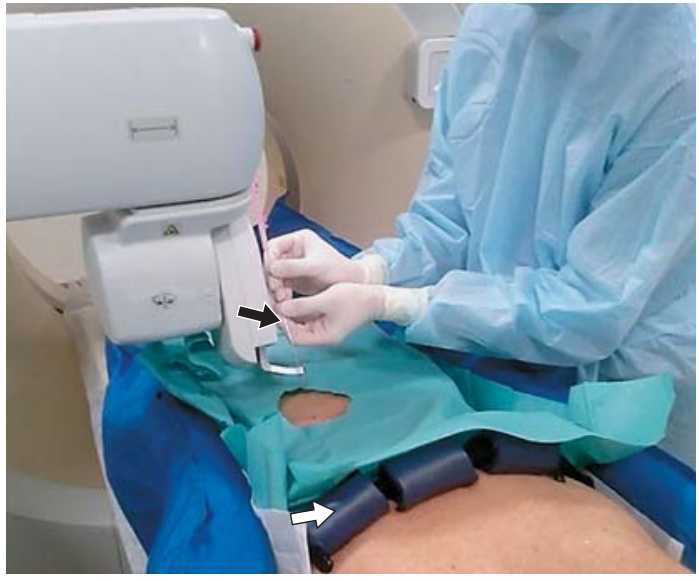

Рис. 5. Фото этапа введения биопсийной иглы. Угол наклона руки манипулятора с установленной иглой (черная стрелка) соответствует расчетным предварительным данным. Пояс-индикатор на уровне живота пациента (белая стрелка).

Fig. 5. Needle placement process. The angle of the endeffector with the inserted needle (black arrow) corresponds to estimated preliminary data. The breath belt is at the level of the patient's abdomen (white arrow).

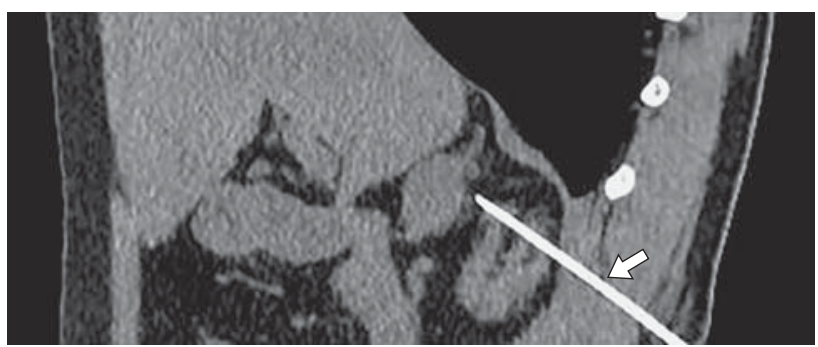

Рис. 6. Компьютерная томограмма брюшной полости пациента M., 54 лет. MPR-реформация в сагиттальной плоскости через ось иглы. Биопсийная игла у края опухоли надпочечника (стрелка).

Fig. 6. Abdominal CT scan of the 54-year-old patient, M.: MPR in sagittal plane through the needle axis. The biopsy needle at the edge of the adrenal gland tumor (arrow).

конец иглы расположен у поверхности образования надпочечника. С помощью биопсийного пистолета осуществлен "выстрел" на глубину 22 мм и забран образец ткани.

По данным гистологического исследования подтвержден метастаз эпителиоидно-клеточной меланомы прогрессирование заболевания.

Как продемонстрировано клиническим наблюдением, использование роботизированной приставки позволило выполнить точное введение биопсийной иглы по сложной траектории на большую глубину и получить информативный образец ткани для гистологического исследования, произведена верификация опухоли, что дало возможность скорректировать лечение пациента.

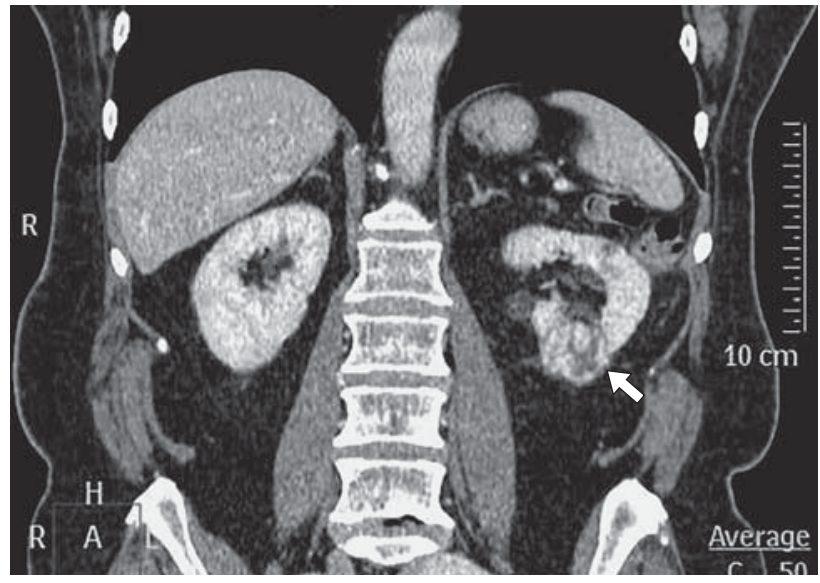

Рис. 7. Компьютерная томограмма брюшной полости пациента С., 81 года. MPR в корональной плоскости через максимальный размер опухоли (стрелка).

Fig. 7. Abdominal CT scan of the 81-year-old patient, C. MPR in coronal plane through the maximum tumor size (arrow).

\section{Клиническое наблюдение 2}

Пациент С., 81 год, диагноз: “светлоклеточный почечно-клеточный рак левой почки (p)T1bNOM0. Лапароскопическая резекция левой почки от 03.07.2012".

В октябре 2018 г. при контрольной КТ живота в нижнем полюсе левой почки выявлено гиперваскулярное образование размерами $25 \times 24$ мм - рецидив рака почки (рис. 7). Учитывая возраст, выраженную сопутствующую патологию, локализацию и размер опухоли, принято решение о проведении криоабляции опухоли почки под КТ-контролем.

После введения в наркоз пациент был расположен на столе томографа и фиксирован с помощью вакуумного матраса в положении "на животе". На остановленном дыхании выполнено сканирование перед манипуляцией. Предварительный анализ изображений КТ с помощью программного обеспечения роботизированной приставки позволил смоделировать зону абляции с оптимальным расположением трех криозондов. Последовательно введены три интродьюсера со стилетами, через один из интродьюсеров осуществлен забор биопсийного материала, далее стилеты заменены на криозонды МКС (рис. 8). Проведено 2 цикла криоабляции с установочной температурой $-195^{\circ} \mathrm{C}$ длительностью 10 и 5 мин с периодом активного отогрева между циклами в течение 10 мин.

При КТ на следующие сутки после операции зона абляции с опухолью аваскулярна, в ее структуре видны дефекты по ходу ранее стоявших криозондов (рис. 9). Изменения в паренхиме почки и периренальной клетчатке, прилежащей к зоне воздействия, выражены незначительно. 

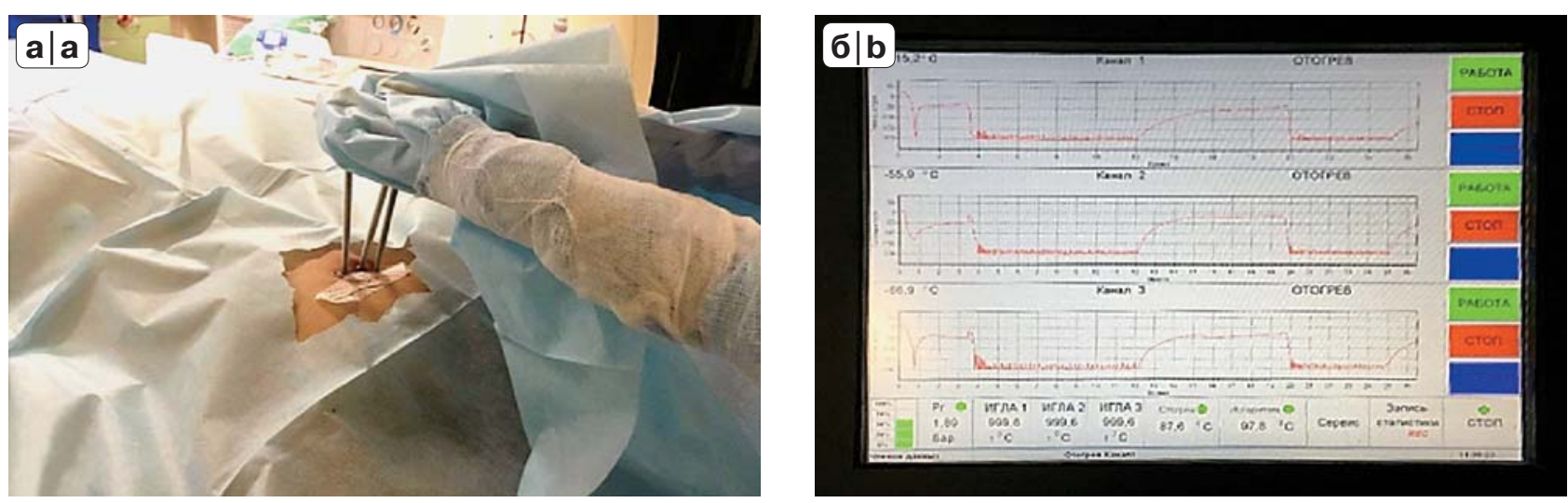

Рис. 8. Фото этапа активной криоабляции опухоли почки. а - расположение криозондов; б - экран МКС с параметрами режима криоабляции.

Fig. 8. The step of cryoablation of renal tumor. a - cryoprobes; b - Medical Cryotherapeutic System screen with cryoablation mode parameters.

Рис. 9. Компьютерная томограмма брюшной полости пациента С., 81 года. MPR в корональной плоскости через максимальный размер опухоли через сутки после криоабляции (стрелка). Пояснение в тексте.

Fig. 9. Abdominal CT scan of the 81-year-old patient, C. MPR in coronal plane through the maximum tumor size in 1 day after cryoablation (arrow). The explanation is in the text.
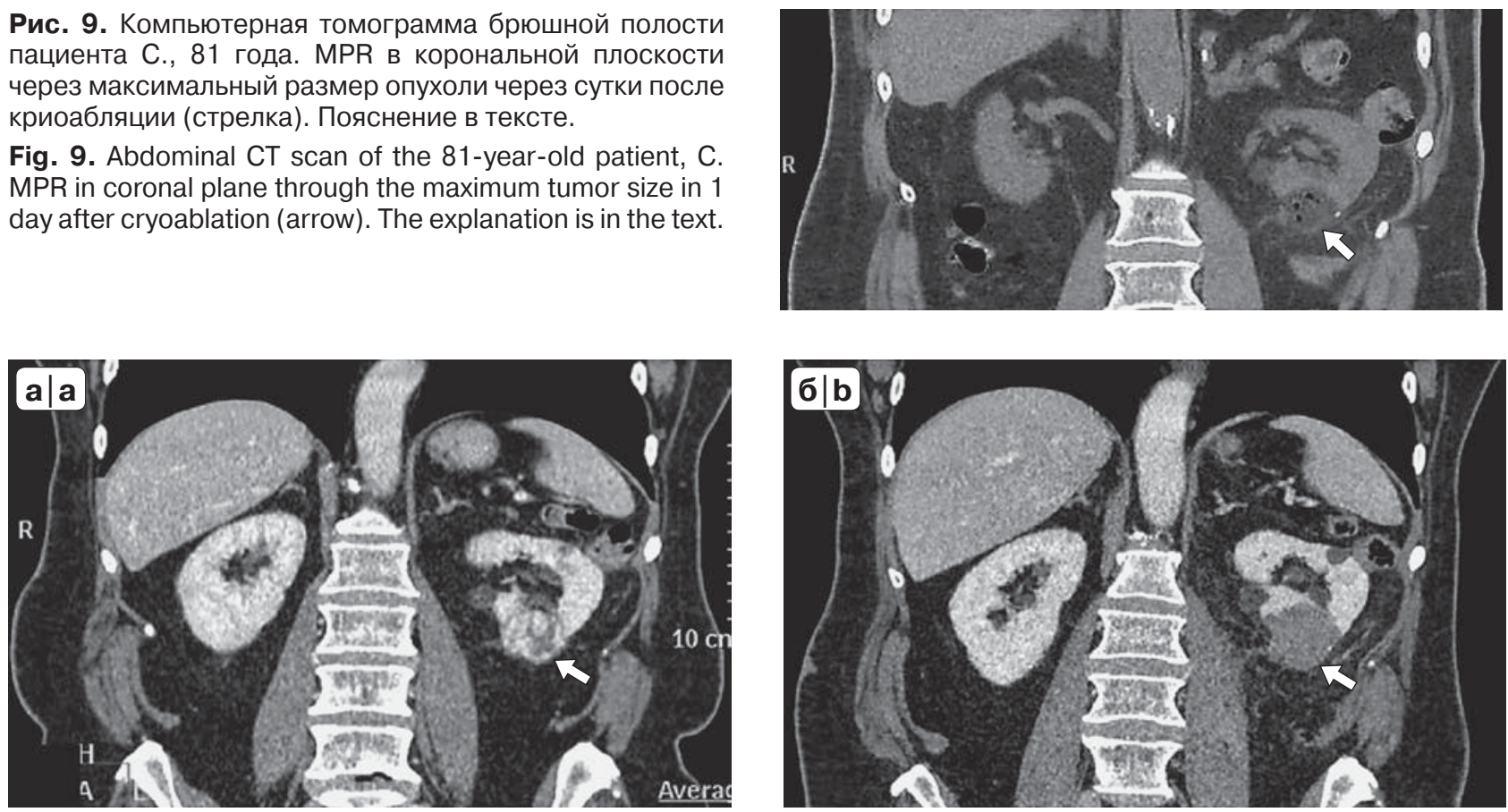

Рис. 10. Компьютерные томограммы брюшной полости пациента С., 81 года. MPR в корональной плоскости. а гиперваскулярная опухоль левой почки до криоаблации (стрелка); б - аваскулярная опухоль в почке через 3 мес после операции (стрелка). Пояснение в тексте.

Fig. 10. Abdominal CT scan of the 81-year-old patient, C.; MPR in coronal plane: a - hypervascular mass in left kidney before cryoablation (arrow); b - avascular mass in the kidney in 3 months after the operation (arrow). The explanation is in the text.

На следующий день после операции пациент отмечал незначительно выраженный дискомфорт в области операции, анализ мочи был нормальным. Пациент был выписан в удовлетворительном состоянии. По данным гистологического исследования подтвержден светлоклеточный почечно-клеточный рак.

Проведено контрольное КТ-сканирование через 3 мес после операции (рис. 10). Как видно на рисунке, на месте ранее существовавшей опухоли в почке имеется аваскулярное образование. Минимальное накопление контрастного препарата, наиболее вероятно, связано с реактивными воспалительными изменениями. Узловых гиперваскулярных образований не выявлено, что указало бы на процедив опухоли.

Приведенное клиническое наблюдение показывает, что использование роботизированной приставки обес- 
печило адекватное планирование и точное позиционирование одновременно трех зондов для оптимальной криодеструкции опухоли почки. Это позволило не только эффективно провести аблятивное вмешательство, но и избежать холодового повреждения прилежащих к опухоли почечной паренхимы, структур почечного синуса, а также и периренальной клетчатки.

\section{Обсуждение}

Миниинвазивные вмешательства, такие как биопсии, дренирования или некоторые лечебные процедуры (микроволновая, крио- и радиочастотная абляция, брахитерапия), как правило, выполняются чрескожно, путем установки иглы в зону интереса (например, в опухоль). Преимуществами такого подхода перед открытыми операциями являются меньшие объем операционной травмы, выраженность болевого синдрома при манипуляции, число послеоперационных осложнений, более короткие сроки госпитализации и восстановления работоспособности $[10,11]$.

Для предоперационной оценки, планирования вмешательства и интраоперационного контроля могут использоваться различные лучевые модальности. Наиболее часто для этих целей применяются УЗИ и КТ. Ультразвуковой метод позволяет проводить мониторинг продвижения иглы в режиме реального времени, не сопровождается ионизирующим излучением $[12,13]$. Ограничение применения метода может быть обусловлено экранированием объекта костными и газосодержащими структурами, глубоким расположением образования, недостаточностью представления о его пространственной конфигурации и взаимоотношении с прилежащими органами.

КТ позволяет эффективно визуализировать большинство образований, при необходимости может сопровождаться контрастным усилением, дает четкое понимание пространственного взаимоотношения инструмента и цели $[12,13]$. К недостаткам метода в первую очередь относится облучение пациента, а также и персонала в случае выполнения вмешательства в режиме КТфлюороскопии. Также минусом является отсутствие возможности визуального контроля в режиме реального времени при последовательном способе проведения операции, а также ограниченность пространства для манипуляции инструментами в гентри КТ при выполнении интервенции под контролем КТ-флюороскопии.

В целом точная установка инструмента в соответствии с запланированной траекторией является критически важным фактором успеха при выполнении чрескожных диагностических и лечебных вмешательств. Для этого могут применяться различные технические решения. Так, в нейрохирургии, требующей особо высокой точности позиционирования инструментов и где имеется возможность жесткой фиксации головы, стереотаксические и роботизированные системы уже используются на протяжении нескольких десятилетий $[14,15]$. Роботизированные приставки для УЗ- и МР-контролируемых вмешательств на предстательной железе являются другим примером подобных широко распространенных систем $[16,17]$.

При КТ-контролируемых операциях на органах груди и живота в последнее время также разрабатываются и используются механические и электронные вспомогательные устройства [18]. Они могут быть как относительно простыми, в виде подвижной фиксирующейся к коже направляющей для иглы определенного диаметра, так и представлять собой сложные многофункциональные роботизированные комплексы, позволяющие планировать и контролировать процесс операции различной сложности.

Для предотвращения движений пациента и смещения цели при интервенционных процедурах рекомендуется применение вакуумных матрасов на этапах предоперационного сканирования и в момент введения иглы [19]. Использование подобных устройств также способствует большему комфорту пациента в сравнении с ситуацией, когда больной длительно расположен на плоском твердом столе компьютерного томографа.

Другим потенциально ограничивающим фактором при чрескожных вмешательствах являются дыхательные экскурсии пациента, приводящее к смещению внутренних органов. Так, если образование в верхушке легкого изменяет свое положение при дыхании незначительно, то при локализации опухоли в наддиафрагмальном отделе может происходить ее смещение на 5 см и более. Поэтому рекомендуют выполнять предоперационное сканирование и введение иглы с фиксированным объемом газовой смеси в легких при их искусственной вентиляции или, если пациент на спонтанном дыхании, ему подаются необходимые команды для задержки дыхания в определенной фазе [20].

В представленном в статье способе при использовании роботизированной приставки факторы движения пациента и дыхания решены именно такими способами - применением вакуумного матраса и пояса для контроля дыхания. Как было продемонстрировано в представленных клинических наблюдениях, использование приставки и вспомогательного оборудования позволяет эффективно решать задачу точного позиционирова- 
ния инструментов при миниинвазивных чрескожных вмешательствах, а также избежать серьезных осложнений.

В то же время существуют на данный момент и нерешенные задачи при применении этого способа. Основной из них представляется необходимость выполнения КТ-сканирования с установленной в теле пациента иглой для верификации соответствия планируемой и фактической траектории введения. Так как этот процесс может занять несколько минут, то, к примеру, в случае вмешательства на легких повышается риск развития осложнений операции. Также при использовании приставки максимальный диаметр рабочего канала на сегодня составляет $11 \mathrm{G}$, что, очевидно, ограничивает применение больших по диаметру инструментов, используемых при криохирургических вмешательствах и при различных видах дренирований и органостомий.

\section{Заключение}

Было продемонстрировано, что способ с использованием роботизированной приставки для малоинвазивных интервенционных вмешательств представляется удачным техническим решением для КТ-контролируемых чрескожных операций, позволяющим эффективно выполнить ряд оперативных этапов, таких как планирование и введение инструмента. Оценка клинической эффективности использования роботизированной приставки в сравнении с традиционными подходами КТ-мониторинга при интервенционных вмешательствах требует дальнейшего изучения на репрезентативных выборках при длительном онкологическом контроле.

\section{Список литературы [References]}

1. Katada K., Kato R., Anno H., Ogura Y., Koga S., Ida Y., Sato M., Nonomura K. Guidance with real-time CT fluoroscopy: early clinical experience. Radiology. 1996; 200: 851-856. http://doi.org/10.1148/radiology.200.3.8756943.

2. Goldberg S.N., Keogan M.T., Raptopoulos V. Percutaneous CT-guided biopsy: improved confirmation of sampling site and needle positioning using a multistep technique at CT fluoroscopy. J. Comput. Assist. Tomogr. 2000; 24: 264-266.

3. Leng S. Radiation Dose in CT-guided Interventional Procedures: Establishing a Benchmark. Radilogy. 2018; 289 (1): 158-159.

http://doi.org/10.1148/radiol.2018181245

4. Bissoli E., Bison L., Gioulis E., Chisena C., Fabbris R. Multislice CT fluoroscopy: technical principles, clinical applications and dosimetry. Radiol. Med. 2003; 106 (3): 201-212.

5. Carlson S.K., Bender C.E., Classic K.L., Zink F.E., Quam J.P., Ward E.M., Oberg A.L. Benefits and safety of CT fluoroscopy in interventional radiologic procedures.
Radiology. 2001; 219 (2): 515-520.

http://doi.org/10.1148/radiology.219.2.r01ma41515.

6. Kimura T., Naka N., Minato Y., Inoue Y., Kimura T., Mawatari H., Yamauchi S., Akira M., Kawahara M. Oblique approach of computed tomography guided needle biopsy using multiplanar reconstruction image by multidetectorrow CT in lung cancer. Eur. J. Radiol. 2004; 52: 206-211. http://doi.org/10.1016/j.ejrad.2004.01.007.

7. Gupta S., Nguyen H.L., Morello F.A. Jr., Ahrar K., Wallace M.J., Madoff D.C., Murthy R., Hicks M.E. Various approaches for CT-guided percutaneous biopsy of deep pelvic lesions: anatomic and technical considerations. Radiographics. 2004; 24: 175-189.

http://doi.org/10.1148/rg.241035063.

8. Cleary K., Melzer A., Watson V., Kronreif G., Stoianovici D. Interventional robotic systems: applications and technology state-of-the-art. Minim. Invasive Ther. Allied. Technol. 2006; 15: 101-113.

http://doi.org/10.1080/13645700600674179.

9. Kettenbach J., Kara L., Toporek G., Fuerst M., Kronreif G. A robotic needle-positioning and guidance system for CTguided puncture: Ex vivo results. Minim. Invasive Ther. Allied. Technol. 2014; 23: 271-278. http://doi.org/10.3109/13645706.2014.928641.

10. Charles E. Ray Jr. Interventional radiology and the care of the oncology patient. Am. Fam. Physician. 2000; 62 (1): 95-102.

11. Ellis L.M., Curley S.A., Tanabe K.K. Radiofrequency Ablation for Cancer: Current Indications, Techniques and Outcomes. New York: Springer. 2004. 307 p.

12. Carberry G.A., Lubner M.G., Wells S.A., Hinshaw J.L. Percutaneous biopsy in the abdomen and pelvis: a stepby-step approach. Abdom. Radiol. 2016; 41 (4): 720-742. http://doi.org/10.1007/s00261-016-0667-1.

13. Sheafor D.H., Paulson E.K., Kliewer M.A., DeLong D.M., Nelson R.C. Comparison of sonographic and CT guidance techniques: does CT fluoroscopy decrease procedure. Am. J. Roentgenol. 2000; 174 (4): 939-942. http://doi.org/10.2214/ajr.174.4.1740939.

14. Spiegel E.A., Wycis H.T., Marks M., Lee A.J. Stereotaxic apparatus for operations on the human brain. Science. 1947; 106 (2754): 349-350.

http://doi.org/10.1126/science.106.2754.349.

15. Kwoh Y.S., Hou J., Jonckheere E.A., Hayati S. A robot with improved absolute positioning accuracy for CT guided stereotactic brain surgery. IEEE Trans. Biomed. Eng. 1988; 35 (2): 153-160. http://doi.org/10.1109/10.1354.

16. Fichtinger G., Burdette E.C., Tanacs A., Patriciu A., Mazilu D., Whitcomb L.L., Stoianovici D. Robotically assisted prostate brachytherapy with transrectal ultrasound guidance - phantom experiments. Brachytherapy. 2006; 5(1): 14-26. http://doi.org/10.1016/j.brachy.2005.10.003.

17. Muntener M., Patriciu A., Petrisor D., Mazilu D., Bagga H., Kavoussi L., Cleary K., Stoianovici D. Magnetic resonance imaging compatible robotic system for fully automated brachytherapy seed placement. Urology. 2006; 68 (6): 1313-1317. http://doi.org/10.1016/j.urology.2006.08.1089.

18. Kettenbach J., Kronreif G. Robotic systems for percutaneous needle-guided interventions. Minim. Invasive Ther. Allied. Technol. 2015; 24 (1): 45-53.

http://doi.org/10.3109/13645706.2014.977299.

19. Bale R.J., Lottersberger C., Vogele M., Prassl A., Czermak B., Dessl A., Sweeney R.A., Waldenberger P., 
Jaschke W. A novel vacuum device for extremity immobilisation during digital angiography: preliminary clinical experiences. Eur. Radiol. 2002; 12: 2890-2894. http://doi.org/10.1007/s00330-002-1492-1.
20. Widmann G., Schullian P., Haidu M., Wiedermann F.J., Bale R. Respiratory motion control for stereotactic and robotic liver interventions. Int. J. Med. Robot. 2010; 6: 343-349. http://doi.org/10.1002/rcs.343.

Для корреспонденции*: Буровик Илья Александрович - 196211, Россия, Санкт-Петербург, ул. Типанова, 7, кв. 53. Тел.: +7-921-961-50-02. E-mail: burovick_ilya@mail.ru

Буровик Илья Александрович - канд. мед. наук, врач-рентгенолог отделения лучевой диагностики, научный сотрудник отделения диагностической и интервенционной радиологии ФГБУ “НМИЦ онкологии имени Н.Н. Петрова” Минздрава России, Санкт-Петербург.

Прохоров Георгий Георгиевич - доктор мед. наук, профессор, ведущий научный сотрудник научного отделения общей онкологии и урологии, врач-онколог хирургического отделения опухолей головы и шеи ФГБУ “НМИЦ онкологии имени Н.Н. Петрова" Минздрава России, директор ООО "Международный Институт криомедицины”, Санкт-Петербург.

Лушина Полина Анатольевна - врач-онколог, уролог хирургического отделения онкоурологии и общей онкологии ФГБУ “НМИЦ онкологии имени Н.Н. Петрова" Минздрава России, Санкт-Петербург.

Васильев Александр Викторович - врач-рентгенолог отделения лучевой диагностики ФГБУ "НМИЦ онкологии имени Н.Н. Петрова" Минздрава России, Санкт-Петербург.

Дегтярёва Екатерина Александровна - врач-ординатор ФГБУ “НМИЦ онкологии имени Н.Н. Петрова" Минздрава России, СанктПетербург.

Contact*: Ilya A. Burovik - 196211, Russia, Saint-Petersburg, Tipanova str., 7 -53. Phone: +7-921-961-50-02. E-mail: burovick_ilya@mail.ru

Ilya A. Burovik - cand. of med. sci., radiologist of Department of Radiology of FSBI "N.N. Petrov NMRC of Oncology" Ministry of Healthcare of the Russian Federation, St. Petersburg.

Georgy G. Prohorov - doct. of med. sci., Professor, oncologist of Department of Head and neck surgical oncology of FSBI "N.N. Petrov NMRC of Oncology" Ministry of Healthcare of the Russian Federation, St. Petersburg.

Polina A. Lushina - oncologist, urologist of Department of Oncourology and General oncology of FSBI "N.N. Petrov NMRC of Oncology" Ministry of Healthcare of the Russian Federation, St. Petersburg.

Aleksandr V. Vasiliev - radiologist of Department of Radiology of FSBI "N.N. Petrov NMRC of Oncology" Ministry of Healthcare of the Russian Federation, St. Petersburg

Ekaterina A. Degtiareva - resident of FSBI "N.N. Petrov NMRC of Oncology" Ministry of Healthcare of the Russian Federation, St. Petersburg.

Поступила в редакцию 11.04.2019. Принята к печати 13.05.2019.
Received on 11.04.2019.

Accepted for publication on 13.05.2019. 